\title{
Adherence and persistence in patients with rheumatoid arthritis at an integrated health system specialty pharmacy
}

\author{
Megan E Peter, PhD; Autumn D Zuckerman, PharmD, BCPS, AAHIVP, CSP; Josh DeClercq, MS; Leena Choi, PhD; \\ Cameron James, PharmD; Katrina Cooper, PharmD, CSP; Jeannie Choi, PharmD; Michael Nadler, PharmD; and \\ S Bobo Tanner, MD
}

\section{What is already known about this subject}

- Disease-modifying anti-rheumatic drugs improve symptoms and delay progression of rheumatoid arthritis,

- Many patients change medication (either "switching" or "cycling" to a different or similar mechanism of action, respectively) during the first year of therapy. but adherence is often sub-optimal.

\section{What this study adds}

- This study assessed switching, cycling, adherence, and persistence to biologic or targeted-synthetic disease modifying anti-rheumatic drugs at an integrated health-system specialty pharmacy.

- Patients with rheumatoid arthritis in our sample demonstrated high medication adherence and persistence and low rates of switching and cycling.

- Findings support evidence that integrated health system specialty pharmacies help patients overcome barriers to medication adherence to persist on therapy.

\author{
Author affiliations \\ Megan E Peter, PhD; Autumn D Zuckerman, \\ PharmD, BCPS, AAHIVP, CSP; Katrina Cooper, \\ PharmD, CSP; Department of Pharmaceutical \\ Services, Vanderbilt University Medical \\ Center, Nashville, TN. Josh DeClercq, \\ MS; Leena Choi, PhD; Department of \\ Biostatistics, Vanderbilt University Medical \\ Center, Nashville, TN. Cameron James, \\ PharmD; Jeannie Choi, PharmD; Sanofi, Inc., \\ Bridgewater Township, NJ. Michael Nadler, \\ PharmD; Lipscomb University College of \\ Pharmacy, Nashville, TN. S Bobo Tanner, \\ MD; Divisions of Rheumatology, Allergy \& \\ Immunology, Vanderbilt University Medical \\ Center, Nashville, TN.
}

AUTHOR CORRESPONDENCE: Megan Peter, 615.936.7889;

megan.peter@vumc.org

J Manag Care Spec Pharm. 2021;27(7):882-90

Copyright (C)2021, Academy of Managed Care Pharmacy. All rights reserved.

\section{ABSTRACT}

BACKGROUND: Disease-modifying anti-rheumatic drugs (DMARDs) improve symptoms and delay progression of rheumatoid arthritis (RA), but adherence is often sub-optimal and many patients change medication (either "switching" to a medication with a different mechanism of action or "cycling" to a medication with the same mechanism of action) during the first year of therapy. Some integrated health-system specialty pharmacies embed pharmacists in clinics to help patients access and adhere to specialty medication.

OBJECTIVE: This study assessed DMARD switching, cycling, adherence, and persistence at an outpatient rheumatology clinic with an integrated health-system specialty pharmacy.
METHODS: We conducted a retrospective cohort study of adults with RA, naïve to biologic or targeted synthetic DMARDs, who filled $\geq 2$ biologic or targeted synthetic DMARD prescriptions within 12 months. Adherence was measured using proportion of days covered (PDC); persistence was computed at 12 months. Univariate analyses compared adherence and persistence between patients with and without a 
medication change. Ordinal logistic regression examined whether PDC was associated with patient age, gender, race, insurance type, and medication change.

RESULTS: We included 772 patients: $79 \%$ female/21\% male, $89 \%$ White $/ 11 \%$ non-White, median age 56 years (interquartile range $=48-63)$. Most patients $(84 \%)$ did not change medication during the study period, $5 \%$ cycled medication one or more times (but did not switch), $9 \%$ switched medication one or more times (but did not cycle), and $2 \%$ of patients both switched and cycled during the study period. Median PDC of the sample was 0.94 and $73 \%$ of patients were persistent. Patients with a medication change had lower PDC than those without ( 0.89 vs $0.95, P=0.004)$, but rate of persistence did not significantly differ between groups ( 77 vs $72 \%, P=0.300$ ). Odds of higher PDC was more likely for men (Odds ratio [OR] $=1.82$, 95\% confidence interval $[\mathrm{CI}]: 1.34-2.48, P<0.001)$ and less likely for patients who changed medication ( $\mathrm{OR}=0.65, \mathrm{Cl}: 0.47-0.91, P=0.011)$; age, race, and insurance type were not significant.

CONCLUSIONS: Patients with RA demonstrated high medication adherence and persistence, and low rates of switching and cycling. Findings support evidence that integrated health-system specialty pharmacies with clinical pharmacists embedded in outpatient clinics help patients overcome barriers to medication adherence to persist on therapy.

Rheumatoid arthritis (RA) is an autoimmune inflammatory arthritis characterized by joint pain and stiffness that can progress to disability and mortality if left untreated. Conventional synthetic disease-modifying anti rheumatic drugs (csDMARDs), such as methotrexate, are first line agents for treating rheumatoid arthritis. ${ }^{1}$ When csDMARDs are unsuccessful, clinical guidelines recommend biologic DMARDs (bDMARDs), which can be characterized as Tumor Necrosis Factor (TNF) Inhibitors (eg, adalimumab) or nonTNF inhibitors (eg, abatacept, tocilizumab). ${ }^{1}$ Recently, a new and growing category of therapy has emerged, targeted synthetic DMARDs (tsDMARDs), which includes medication such as tofacitinib.

Adherence and persistence to DMARD therapy are necessary for achieving therapeutic benefits, with the goal of disease remission or low disease activity. ${ }^{2,3}$ Adherence, which is the extent to which a patient takes a medication as prescribed, is often calculated as medication possession ratio (MPR) or proportion of days covered (PDC) or as the proportion of patients achieving a threshold of MPR or PDC (eg, $\geq 0.80$ ) over a specified timeframe., ${ }^{4,5}$ Medication persistence, which refers to the duration of continuous therapy use, is often calculated as the number of days from initiation of therapy to discontinuation or as the proportion of patients continuing therapy for a predefined duration without a predefined gap in treatment (eg, continuation of therapy for one-year without a gap of 60 days). ${ }^{4-8}$ Adherence to bDMARD therapy is often suboptimal, ranging from $0.52-0.88$ as measured by MPR. ${ }^{9-11}$ Similarly, persistence is often low, and studies have reported between $43 \%-68 \%$ of patients discontinue the initial bDMARD within the first year of therapy. ${ }^{9,11}$

The American College of Rheumatology's 2015 guidelines recommend selecting and adjusting treatments until patients achieve low RA disease activity, with limited evidence to support a specific treatment algorithm. ${ }^{1}$ When the initial bDMARD is ineffective, providers decide whether to change to a medication with the same mechanism of action (MoA), known as "cycling," or to a different MoA, known as "switching." Recent data found that cycling is more common than switching, ${ }^{12}$ but switchers are more likely to persist on the second therapy than cyclers. ${ }^{13}$ Changes in therapy can be challenging to navigate as many insurers require extensive prior authorization and step-therapy requirements and financial assistance is often needed for patients to afford their medication. ${ }^{14}$

The objectives of this study were to assess medication adherence, persistence, switching, and cycling in adults with RA during their first year of bDMARD or tsDMARD therapy. Eligible patients received care at an outpatient rheumatology clinic and filled medication at an integrated health-systems specialty pharmacy. Some integrated specialty pharmacies embed pharmacists in clinics to provide detailed medication counseling and monitoring and to dispense medication from an internal pharmacy. This model has demonstrated benefits such as timely access to medication, low patient out-of-pocket medication costs, and high patient adherence and persistence to therapy. ${ }^{15-19}$ Understanding the pharmacist's role and patient outcomes in this model is needed to inform best practices in specialty pharmacy for patients with RA.

\section{Methods}

\section{SETTING}

The study was conducted at Vanderbilt University Medical Center (VUMC), a large academic health system in the Southeast United States within two outpatient adult rheumatology clinics. VUMC has an integrated specialty pharmacy (Vanderbilt Specialty Pharmacy) that dispenses self-administered specialty medication. Clinical specialty pharmacists were embedded into the first Rheumatology Clinic in July 2013 and the second Rheumatology Clinic in January 2015. Pharmacists work alongside providers to manage patient access to therapy, conduct medication counseling, and 


\section{TABLE 1 Included Medications By Mechanism of Action and Frequency of Prescriptions (Total $n=7,536$ )}

\begin{tabular}{|c|c|c|c|c|}
\hline $\begin{array}{l}\text { Mechanism of Action } \\
\text { (MoA) }\end{array}$ & $\begin{array}{l}\text { MoA } \\
\text { N (\%) }\end{array}$ & $\begin{array}{l}\text { Brand } \\
\text { name }\end{array}$ & Generic name & $\begin{array}{c}\text { Prescriptions } \\
\mathbf{N}(\%)\end{array}$ \\
\hline \multirow{4}{*}{$\begin{array}{l}\text { Biologic DMARD (bDMARD): } \\
\text { Tumor Necrosis Factor } \\
\text { (TNF) inhibitors }\end{array}$} & \multirow{4}{*}{$5,674(75)$} & Cimzia & Certolizumab pegol & $251 \quad(3)$ \\
\hline & & Enbrel & Etanercept & $2,485 \quad(33)$ \\
\hline & & Humira & Adalimumab & $2,732(36)$ \\
\hline & & Simponi & Golimumab & $206 \quad(3)$ \\
\hline \multirow{2}{*}{$\begin{array}{l}\text { Biologic DMARD (bDMARD): } \\
\text { Interleukin-6 (IL-6 inhibitors) }\end{array}$} & \multirow{2}{*}{$207 \quad(3)$} & Actemra & Tocilizumab & $207 \quad(3)$ \\
\hline & & Kevzara & Sarilumab & $0 \quad(0)$ \\
\hline $\begin{array}{l}\text { Biologic DMARD (bDMARD): } \\
\text { T cell inhibitor }\end{array}$ & $813(11)$ & Orencia & Abatacept & $813(11)$ \\
\hline $\begin{array}{l}\text { Biologic DMARD (bDMARD): } \\
\text { Interleukin-1 (IL-1) inhibitor }\end{array}$ & $19(<1)$ & Kineret & Anakinra & $19(<1)$ \\
\hline \multirow{2}{*}{$\begin{array}{l}\text { Targeted synthetic DMARD } \\
\text { (tsDMARD): Janus kinase } \\
\text { (JAK) inhibitor }\end{array}$} & \multirow{2}{*}{$823(11)$} & Xeljanz & Tofacitinib & $823(11)$ \\
\hline & & Olumiant & Baricitnib & $0 \quad(0)$ \\
\hline
\end{tabular}

monitor patient response and safety on therapy. The pharmacy team frequently assesses adherence barriers or need to change medication because of adverse effects, treatment ineffectiveness, insurance changes, cost barriers, or patient preference.

\section{DATA COLLECTION AND MANAGEMENT}

Data were collected from electronic health records and pharmacy claims at VUMC and Vanderbilt Specialty Pharmacy. From electronic health records, we extracted patient date of birth, gender, and race. From pharmacy claims, we collected the name, days' supply, insurance type, and dispense date of each eligible medication dispensed from Vanderbilt Specialty Pharmacy during the study period. Data were stored in Research Electronic Data Capture (REDCap), a HIPAA-secure web-based tool for data collection and management. ${ }^{20}$

\section{STUDY DESIGN}

We conducted a retrospective cohort study of adults with RA who received care from VUMC and filled prescriptions through Vanderbilt Specialty Pharmacy. Data were collected from July 1, 2013 through June 30, 2018 for patients treated at the first clinic, and from January 2, 2015 through June 30, 2018 for patients treated at the second clinic. The study was approved by Vanderbilt University Institutional Review Board.

\section{SAMPLE}

We included patients who met the following criteria: (1) diagnosed with RA as indicated by an ICD-10-CM code diagnosis of M05 or M06 in the electronic health record; (2) two or more bDMARD or tsDMARD prescriptions (prescribed by a healthcare provider within one of the rheumatology clinics) dispensed from Vanderbilt Specialty Pharmacy in a twelve-month period; (3) aged 18 years or older at initial dispense date (ie, index date) within the study period; (4) treatment naïve at index date, defined as the absence of a BDMARD or tsDMARD prescription dispensed from VSP within 120 days before index date; and (5) index date on or before June 30, 2017. Included medications, sorted by their MoA, are shown in Table 1. We excluded patients who received only sample medications during the twelve-month observation period.

\section{ANALYSIS}

Descriptive statistics were used to summarize characteristics of patients included in the study. Frequencies and percentages were calculated for categorical variables; the mean, median, standard deviation (SD), and interquartile range (IQR) were computed for continuous variables. Time to first switch and time to first cycle were computed for the subsets of patients who switched and patients who cycled, respectively. Data were analyzed using the programming language $\mathrm{R}$ version 3.6.2. The following analyses were conducted to assess the study outcomes:

Rates of Switching and Cycling. We estimated the rate of any switch or cycle between medications, defined as follows: Switching was defined as a change from one bDMARD/tsDMARD to another bDMARD/tsDMARD with a different MoA within 12-months of index date (eg, switching from a TNF inhibitor to a non-TNF inhibitor). Cycling was defined as a change from one bDMARD/tsDMARD to another bDMARD/tsDMARD with the same MoA within 12 months of index date (eg, changing from a TNF inhibitor to another TNF inhibitor).

Adherence. Adherence was calculated using PDC, which is an indirect metric of medication administration computed from claims data using dates and days' supply of each dispense. Because we did not encounter any instances of polypharmacy (ie, patients prescribed two or more medications concurrently), in the occurrence of a switch or cycle, excess supply of the initial drug was assumed to be discarded and carryover of drug supply was set to zero in the PDC calculation. We 


\section{TABLE 2 Characteristics of the Sample $(n=772)$}

\begin{tabular}{|c|c|c|c|c|c|}
\hline Characteristic & $\mathbf{n}$ & $\%$ & Characteristic & $\mathbf{n}$ & $\%$ \\
\hline \multicolumn{3}{|l|}{ Gender } & \multicolumn{3}{|l|}{ Number of times patient cycled } \\
\hline Male & 165 & 21 & 0 & 718 & 93 \\
\hline Female & 607 & 79 & 1 & 53 & 7 \\
\hline \multicolumn{3}{|l|}{ Race } & 3 & 1 & $<1$ \\
\hline White & 686 & 89 & \multicolumn{3}{|l|}{ Persistent at end of study period ${ }^{b}$} \\
\hline Black or African American & 63 & 8 & Non-persistent & 210 & 27 \\
\hline Other & 11 & 1 & Persistent & 562 & 73 \\
\hline Unknown & 12 & 2 & \multicolumn{3}{|l|}{ Persistent throughout entire index yearc } \\
\hline \multicolumn{3}{|l|}{ Insurance type } & Non-persistent & 281 & 36 \\
\hline Commercial & 464 & 60 & Persistent & 491 & 64 \\
\hline $\begin{array}{l}\text { Government (i.e., Medicare, Medicaid, or } \\
\text { Tricare) }\end{array}$ & 291 & 38 & \multicolumn{3}{|c|}{ Restarts (after prior non-persistence in index period) ${ }^{d}$} \\
\hline Multiple & 17 & 2 & 0 & 185 & 66 \\
\hline \multicolumn{3}{|c|}{ Switching and cycling in 12-months post-index ${ }^{a}$} & 1 & 89 & 32 \\
\hline No switch or cycle & 648 & 84 & 2 & 7 & 2 \\
\hline Cycle only & 39 & 5 & & Median & \\
\hline Switch and cycle & 15 & 2 & & [IQR] & Mean \pm SD \\
\hline Switch only & 70 & 9 & Age at index date, years & $\begin{array}{c}56 \\
{[48-63]}\end{array}$ & $55 \pm 13$ \\
\hline \multicolumn{3}{|l|}{ Number of times patient switched } & \multicolumn{3}{|l|}{ Proportion of days covered (PDC) } \\
\hline 0 & 687 & 89 & \multirow{2}{*}{ Calculated with the primary definition } & \multirow{2}{*}{$\begin{array}{c}0.94 \\
{[0.81-1.00]}\end{array}$} & \multirow{2}{*}{$0.86 \pm 0.18$} \\
\hline 1 & 72 & 9 & & & \\
\hline 2 & 11 & 1 & \multirow[t]{2}{*}{ Calculated with a fixed interval } & \multirow{2}{*}{$\begin{array}{c}0.87 \\
{[0.61-0.98]}\end{array}$} & \multirow[t]{2}{*}{$0.76 \pm 0.26$} \\
\hline 3 & 2 & $<1$ & & & \\
\hline
\end{tabular}

aswitch refers to changing to a medication with a different mechanism of action. Cycle refers to changing to a medication with the same mechanism of action.

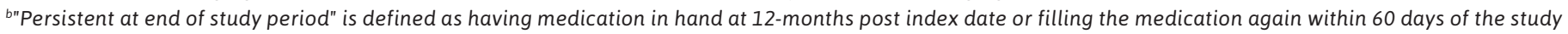
period end.

"Persistent throughout entire index year" is defined as the absence of any 60-day medication gap during the 12-month window.

${ }^{d}$ Restarts refer to the number of times a patient resumed therapy after a gap of $\geq 60$ days (of 281 patients with a gap of $\geq 60$ days during the 12 -month window).

computed each patient's PDC between date of initial medication dispense and date of last dispense (called last fill end rule). ${ }^{21}$ We also reported the number and percent of patients with $\mathrm{PDC} \geq 0.80$. As an exploratory measure, we computed a fixed interval PDC from index date through 12 months, for a denominator of 365 days.

Persistence. Medication persistence typically refers to the duration in which a patient continues treatment without a non-permissible gap, discontinuation, or change in therapy; persistence can also be reported as the proportion of patients who continue on therapy for a specified time frame without a predefined gap (eg, for a one-year duration, without a gap of $\geq 30,45,60$, or 90 days). ${ }^{4,5}$ We used a 60-day gap to measure persistence. ${ }^{13,22}$ Patients were considered persistent at the end of the study period if: (1) the last covered date occurred after the 12-month post-index date, or (2) the first fill after the study end date occurred within 60 days of last date without medication in hand (ie, the absence of a 60-day gap at the end of the study period). As a secondary measure, we computed persistence over the course of the entire study period, defined as the absence of any $\geq 60$ day gap in therapy in the 12 months following index date; of these, we calculated the number of patients who restarted medication after a 60-day gap in therapy.

Univariate Analyses. Univariate analyses were performed to assess whether adherence and persistence were associated with whether a patient switched and/or cycled medication. Pearson's chi-squared test was used to compare the rate of persistence at end of study period between patients who switched and/or cycled medication versus those with no 


\section{FIGURE 1 Medication That Patients Switched or Cycled To and From in a 12-Month Period}



Cycling from a TNF inhibitor to another TNF inhibitor occurred 56 times. The most common switch was from TNF inhibitor to T-cell inhibitor $(n=38)$. Median time to first cycle $(n=54)$ was 120 days after first dispense $(I Q R=77-216$, Mean $=149, S D=94)$. Median time to first switch $(n=85)$ was 186 days $(I R Q=105-256, M e a n=179$, $S D=88)$ after initial medication dispense.

medication change during the study period. Wilcoxon test was used to compare PDC of patients who switched and/or cycled medication versus those without a switch or cycle during the study period.

Multivariable Analysis. We performed ordinal logistic regression analysis to examine whether any switch or cycle was associated with PDC after adjusting for the following covariates: age, gender, race (White vs non-White), insurance type (government vs commercial vs multiple).

\section{Results}

\section{SAMPLE CHARACTERISTICS}

We included 772 patients, most of whom were female (79\%) and White (89\%), with median age of 56 years (IQR=48-63). Sample demographics are shown in Table 2. Patients' number of dispenses during the 12-month period ranged from 2 to 17 . Of the 7,536 medication dispenses to all patients over a 12 -month period, $75 \%$ were TNF inhibitors and $25 \%$ were other MoAs; the most common medications dispensed were adalimumab (36\%) and etanercept (33\%). Frequencies of medications dispensed are also shown in Table 1.

\section{SWITCHING AND CYCLING}

As shown in Table 2, most patients (84\%) did not switch or cycle during the study period. Five percent cycled medication one or more times (but did not switch), $9 \%$ of patients switched medication one or more times (but did not cycle), and $2 \%$ of patients both switched and cycled during the study period. Eleven patients switched therapy twice, two patients switched three times, one patient cycled three times. In total, patients in the sample experienced 56 cycles and 100 switches. Figure 1 shows which MoA patients switched or cycled to and from. Most common switches were from a TNF inhibitor to T-cell inhibitor $(\mathrm{n}=38)$ and TNF inhibitor to Janus kinase (JAK) inhibitor $(n=22)$. Median time to first cycle was 120 days (IQR $=77-216$, Mean $=194$, $\mathrm{SD}=94, \mathrm{n}=54$ ) and median time to first switch was 186 days $(\mathrm{IQR}=105-256$, Mean $=179, \mathrm{SD}=88, \mathrm{n}=85)$.

\section{ADHERENCE AND PERSISTENCE}

Adherence and persistence are summarized in Table 2 . The median PDC in the sample was $0.94(\mathrm{IQR}=0.81-1.00$, Mean $=0.86, \mathrm{SD}=0.18$ ). Seventy-six percent of patients had $\mathrm{PDC} \geq 80 \%$. In the PDC calculation using a fixed interval, median $\mathrm{PDC}$ was $0.87(\mathrm{IQR}=0.61-0.98$, Mean $=0.76, \mathrm{SD}=0.26)$. 


\section{TABLE 3} Adherence and Persistence Between Patients With Any
Switch or Cycle Versus No Switch or Cycle During the
Study Period

\begin{tabular}{|c|c|c|c|c|c|}
\hline & \multicolumn{2}{|c|}{$\begin{array}{l}\text { Any switch or cycle } \\
\qquad(n=124)\end{array}$} & \multicolumn{2}{|c|}{$\begin{array}{l}\text { No switch or cycle } \\
(n=648)\end{array}$} & \multirow[b]{2}{*}{ P value ${ }^{a}$} \\
\hline & n & $\%$ & n & $\%$ & \\
\hline \multicolumn{6}{|c|}{ Persistent at end of study period } \\
\hline Non-persistent & 29 & 23 & 181 & 28 & \multirow{2}{*}{0.3} \\
\hline \multirow[t]{2}{*}{ Persistent } & 95 & 77 & 467 & 72 & \\
\hline & Median [IQR] & Mean \pm SD & Median [IQR] & Mean \pm SD & P value $^{\text {b }}$ \\
\hline $\begin{array}{l}\text { Proportion of days covered } \\
\text { (PDC) }\end{array}$ & $\begin{array}{c}0.89 \\
{[0.79-0.98]}\end{array}$ & $0.83 \pm 0.20$ & $\begin{array}{c}0.95 \\
{[0.81-1.00]}\end{array}$ & $0.87 \pm 0.18$ & 0.004 \\
\hline
\end{tabular}

aPearson's Chi-Squared Test

bWilcoxon Test.

As shown in Table 2, $73 \%$ of patients were persistent at the end of the study period and $64 \%$ of patients were persistent throughout the entire observation period. Of the 281 patients with non-persistence (ie, gap of $\geq 60$ days) during the study period, 96 restarted their medication after the gap; of these, 7 patients restarted medication twice after separate gaps in therapy.

Table 3 compares patients with any switch and/or cycle $(n=124)$ to those without a switch or cycle $(n=648)$. Median PDC was lower for patients with a switch and/or cycle than those without (0.89 vs $0.95, \mathrm{P}=0.004)$, but the rate of persistence to the end of the study period did not differ between the two groups (77\% vs $72 \%$, $\mathrm{P}=0.300$ ).

\section{MULTIVARIABLE ANALYSIS}

Table 4 shows the results of ordinal logistic regression analysis for PDC in the 12-month study period. Holding all other variables constant, the results indicate male patients were $82 \%$ more likely to have a higher PDC than female patients (odds ratio $[\mathrm{OR}]=1.82,95 \%$ confidence interval [CI]: 1.34 to 2.48 , $\mathrm{P}<0.001)$. Additionally, patients who switched or cycled were $35 \%$ less likely to have higher adherence than those without a switch or cycle $(\mathrm{OR}=0.65$, 95\% CI: 0.47 to $0.91, P=0.011$ ).

\section{Discussion}

In this sample of adult patients with RA during the first year of bDMARD or tsDMARD therapy, rates of medication switching and cycling were low and adherence and persistence to therapy were high. This study is the first to our knowledge to explore rates of switching and cycling at a health system with an integrated specialty pharmacy with clinical pharmacists embedded in the outpatient clinics.

We found high adherence to medication in our sample, with a median PDC of 0.94. Moreover, three-quarters of the sample ( $76 \%$ of patients) achieved PDC greater than or equal to 0.80 . Findings are consistent with previous literature which show high medication adherence in multiple sclerosis, oncology, and pulmonary clinics at integrated specialty pharmacies. ${ }^{15,17,23}$

We also found high medication persistence, with $73 \%$ of patients persistent 12 months after their first dose of bDMARD or tsDMARD.
Using a persistence definition of the absence of any gap of $\geq 60$ days over the study period, persistence was lower $(64 \%)$, indicating that patients may experience one or more extended gap periods within their first year of therapy. Both methods of calculating persistence resulted in higher rates than previous studies, which range from $44 \%$ to $67 \%$ at twelve months.

Adherence to bDMARD and tsDMARD therapy is vital to achieving clinical benefit from these expensive therapies., ${ }^{3,24,25}$ Higher adherence is associated with fewer inpatient and outpatient healthcare utilization. ${ }^{24}$ Thus, the Pharmacy Quality Alliance (PQA) recommends an adherence threshold of $\geq 0.80$ for adult patients using non-infused biologic medications used to treat rheumatoid arthritis. ${ }^{26}$ Barriers to treatment adherence include high treatment costs, injectable medication administration, variable dosing schedules (which may be daily, weekly, every other week, monthly, etc.), adverse reactions and side effects, and precautions (eg, serious infections) that may require patients to temporarily discontinue therapy.

All biologic DMARDS carry risk of potential side effects and treatment inefficacy, which warrant a change in therapy according to ACR guidelines. ${ }^{1,27,28}$ Some studies suggest that cycling through TNF inhibitors is more common than switching. In one study of 631 patients, the first line therapy was TNF inhibitors for 535 (84.4\%) of patients, and 163 (25.8\%) changed to second use targeted therapy during the study period: $60.7 \%$ to another TNF inhibitor, $39.3 \%$ to a different MoA. ${ }^{12}$ We found lower rates of switching and cycling in our sample: $84 \%$ of the sample did not switch or cycle during the twelve-month period, $9 \%$ of patients switched, 5\% cycled, and $2 \%$ both switched and cycled. At the outpatient rheumatology 


\section{TABLE 4 Ordinal Logistic Regression Assessing Characteristics Associated With Proportion of Days Covered (PDC)}

\begin{tabular}{l|c|c|c}
\hline \multicolumn{1}{c|}{ Predictor } & $\begin{array}{c}\text { Odds ratio } \\
\text { (OR) }\end{array}$ & $\begin{array}{c}\text { 95\% confidence } \\
\text { interval (Cl) }\end{array}$ & P value \\
\hline Age (per 10 years) & 1.04 & $0.93-1.16$ & 0.521 \\
\hline Male gender (ref = female) & 1.82 & $1.34-2.48$ & $<0.001$ \\
\hline White race (ref = non-white) & 0.95 & $0.64-1.42$ & 0.807 \\
\hline Government insurance (ref=commercial) & 1.20 & $0.90-1.60$ & 0.220 \\
\hline Multiple insurers (ref=commercial) & 0.66 & $0.28-1.55$ & 0.337 \\
\hline Any switch/cycle (ref = no change) & 0.65 & $0.47-0.91$ & 0.011 \\
\hline
\end{tabular}

clinics, clinical pharmacists-who are embedded in clinics as members of the healthcare team-collaborate with physicians to select appropriate therapy for patients. After the decision to treat, pharmacists review patients' insurance formulary to determine if they meet insurance requirements (eg, step therapy) to start biologic therapy and notify the provider of the formulary-preferred medication option(s). If patients have a contraindication to the preferred medication or if a non-preferred medication is more clinically appropriate, pharmacists work with providers to gather documents to submit an appeal to insurance to approve coverage of the medication. We hypothesize that integrated clinical pharmacists may be better able to help physicians select the most clinically and financially appropriate initial therapy upfront, which might explain low rates of medication changes needed in the first year of therapy.

The pharmacy team within the integrated pharmacy, which includes clinical pharmacists and pharmacy technicians, also monitor and address patient barriers to treatment adherence. They ensure patients can access, afford, and administer therapy as prescribed. The pharmacy team navigates insurance approval (eg, prior authorizations, appeals) and financial assistance enrollment; conducts medication counseling; and performs monthly monitoring assessments for adherence, adverse effects, and healthcare utilization. All activities are documented in the electronic health record, allowing real-time communication between pharmacists and prescribers. Our findings contribute to a growing body of literature on treatment patterns and patient outcomes in patients with RA and highlight the value of the integrated specialty pharmacy for clinics and patients. Data suggest the integrated care model allows high-touch care that effectively prevents, identifies, and resolves barriers to adherence and persistence. Moreover, with clinical pharmacists as embedded members of the healthcare team, patients have access to medication counseling and frequent monitoring, so patients in this setting may be more likely to persist on therapy for a longer duration.

Adherence was lower in patients who switched or cycled compared with the no switch/cycle group (0.89 vs 0.95 PDC), but treatment persistence at 12 months did not differ between groups. One plausible explanation for this could be that the turbulence precipitating the switch or cycle (be it adverse effects or treatment inefficacy) means patients are less likely to remain as vigilant when refilling their medication. However, adherence in the switch or cycle group still exceeds the PQA threshold for adherence and is higher than many previous studies. Findings suggest that the high-touch integrated care model promotes high adherence and persistence, as the pharmacists are quick to identify necessary changes in therapy without delay. At monthly refill calls, the pharmacy technician asks patients if they have missed any doses or incurred any adverse reactions to their medication; if so, pharmacists are flagged to intervene by reviewing the patient's chart, contacting the patient, and alerting the prescriber if needed. As members of the healthcare team, integrated clinic pharmacists can communicate with healthcare providers in real time to address patients' healthcare needs, which may result in a therapy change or dose change. Further research is needed to better understand the reasons for discontinuation and extended gaps in therapy and to explore how pharmacists can assist patients and providers in identifying the need to stop or change therapy due to safety or efficacy concerns.

We calculated adherence using PDC, which is more conservative than MPR for measuring adherence from administrative data as it only accounts for the days when the patient could have conceivably taken the medication. Comparing adherence and persistence across studies is limited by inconsistent reporting and heterogeneous methods for calculating these metrics, including data source and quality, sample size and characteristic, and definitions of metrics. ${ }^{6}$ We selected our adherence and persistence calculation methods to minimize overlap between the calculations: adherence is measured during the time that the patient fills medication, whereas persistence reflects whether the patient has continued with the medication up to one year, independent of how 
adherent they were with their medication regimen. As a sensitivity analysis, we also reported PDC using a 365-day denominator (called a fixed interval), and persistence as the absence of any $\geq 60$-day gap during the twelve-month window, to allow our results to be compared with studies that used this definition..$^{13,22}$ As expected, the PDC with a fixed interval was lower than that of varying intervals (Table 2); this is because the denominator is larger for the PDC with a fixed interval calculation, as the fixed interval may include an extended time period for some patients who were lost to follow-up for various reasons (eg, death, transferring medication to another pharmacy).

We also found a significant association between gender and adherence in multivariable analysis, with male patients $82 \%$ more likely to have a higher PDC than female patients; however, race, age, and insurance type were not associated with PDC. Previous research exploring sociodemographic correlates of medication adherence have produced inconsistent results. Some studies have found that patients who are older age, male gender, and White (compared with African American) have higher medication adherence. ${ }^{29,30}$ Others have found non-significant associations between age and gender with adherence. ${ }^{31}$ Given that RA predominately affects women, further research is warranted-namely, research using prospective study design-to explore psychosocial reasons why women may be less likely to be adherent to therapy than men, accounting for other characteristics (eg, disease severity, presence of comorbidities, and perceived treatment efficacy) that may be associated with adherence.

\section{LIMITATIONS}

These results were obtained from pharmacy claims data, an indirect measure of medication-taking behavior. As such, these data possess inherent limitations in calculations of adherence and persistence, as medication dispenses do not incorporate reasons for gaps in therapy such as patient death, transfer to another healthcare provider or pharmacy, intentional therapy holds, or use of sample medications. To reduce risk of inaccuracies, we excluded patients who received only sample medications during the twelve-month observation period, as these patients may transfer prescriptions to an external pharmacy after an initial sample. Patients with fewer than two medication dispenses during the study period were excluded in an attempt to remove patients who transfer medications to a non-integrated specialty pharmacy early in their treatment course.

Data included extractable information for prescriptions at a single pharmacy that dispenses only selfadministered medications, so we could not account for instances when patients transitioned to or from an infused drug; thus, our rate of persistence and adherence could be underestimated.

Our analysis included a limited number of demographic characteristics that could be extracted from electronic health records with high accuracy and completeness of data: age, gender, and race. We expect that other demographic, psychosocial, or health-related characteristicsbeyond what can be extracted from retrospective pharmacy claims dataare associated with patient adherence and persistent to therapy. Future work is warranted to further explain and predict patient characteristics associated with treatment behaviors and health outcomes. Moreover, future work is needed to explore how changes in inflammatory markers and other clinical assessments influence patient adherence, persistence, and therapy changes. Lastly, the findings from this study are specific to the practice at a single healthcare system and integrated specialty pharmacy, and results may not be generalizable to other settings.

\section{Conclusions}

Patients with RA who received care at an outpatient rheumatology clinic and filled bDMARD or tsDMARD medication at an integrated health-systems specialty pharmacy demonstrated high adherence and persistence to therapy and low rates of switching and cycling. Findings support evidence that integrated health-systems specialty pharmacies with pharmacists embedded into clinics help patients overcome barriers to medication adherence to persist on therapy.

\section{DISCLOSURES}

This study was funded by Sanofi, Inc. James and J. Choi were employed by Sanofi, Inc., at the time of this study. Peter, Zuckerman, DeClercq, L. Choi, and Tanner, received research funding from Sanofi, Inc., for work on this study. Tanner has also received advisory board/speaker bureau fees from Pfizer, Regeneron, and Sanofi-Aventis.

This study was presented as a poster at AMCP Nexus in October 2019 at National Harbor, MD.

\section{REFERENCES}

1. Singh JA, Saag KG, Bridges SL, Jr., et al. 2015 American College of Rheumatology Guideline for the Treatment of Rheumatoid Arthritis. Arthritis Care Res (Hoboken). 2016;68(1):1-25.

2. Li L, Cui Y, Yin R, et al. Medication adherence has an impact on disease activity in rheumatoid arthritis: a systematic review and meta-analysis. Patient Prefer Adherence. 2017;11:1343-56. 
3. Bluett J, Morgan C, Thurston L, et al. Impact of inadequate adherence on response to subcutaneously administered anti-tumour necrosis factor drugs: results from the Biologics in Rheumatoid Arthritis Genetics and Genomics Study Syndicate cohort. Rheumatology (Oxford). 2015;54(3):494-99.

4. Cramer JA, Roy A, Burrell A, et al. Medication compliance and persistence: terminology and definitions. Value Health. 2008;11(1):44-47.

5. Murage MJ, Tongbram V, Feldman SR, et al. Medication adherence and persistence in patients with rheumatoid arthritis, psoriasis, and psoriatic arthritis: a systematic literature review. Patient Prefer Adherence. 2018;12:1483-503.

6. Blum MA, Koo D, Doshi JA. Measurement and rates of persistence with and adherence to biologics for rheumatoid arthritis: a systematic review. Clin Ther. 2011;33(7):901-13.

7. Neubauer S, Cifaldi M, Mittendorf T, et al. Biologic TNF inhibiting agents for treatment of rheumatoid arthritis: persistence and dosing patterns in Germany. Health Econ Rev. 2014;4(1):32.

8. Wu N, Lee YC, Shah N, et al. Cost of biologics per treated patient across immune-mediated inflammatory disease indications in a pharmacy benefit management setting: a retrospective cohort study. Clin Ther. 2014;36(8):1231-1241, 1241. e1231-3.

9. Curkendall S, Patel V, Gleeson M, et al. Compliance with biologic therapies for rheumatoid arthritis: do patient outof-pocket payments matter? Arthritis Rheum. 2008;59(10):1519-26.

10. Khilfeh I, Guyette E, Watkins J, et al. Adherence, Persistence, and Expenditures for High-Cost Anti-Inflammatory Drugs in Rheumatoid Arthritis: An Exploratory Study. J Manag Care Spec Pharm. 2019;25(4):461-67.

11. Strand V, Miller P, Williams SA, et al. Discontinuation of Biologic Therapy in Rheumatoid Arthritis: Analysis from the Corrona RA Registry. Rheumatol Ther. 2017;4(2):489-502.
12. Sullivan E, Kershaw J, Blackburn S, et al. Biologic Disease-Modifying Antirheumatic Drug Prescription Patterns for Rheumatoid Arthritis Among United States Physicians. Rheumatol Ther. 2020;7(2):383-400.

13. Chastek B, Chen CI, Proudfoot C, et al. Treatment Persistence and Healthcare Costs Among Patients with Rheumatoid Arthritis Changing Biologics in the USA. Adv Ther. 2017;34(11):2422-35.

14. Cocohoba J, Pohlman B, Tran JS, et al. Modeling specialty medicine access: Understanding key health system processes and players. J Am Pharm Assoc (2003). 2019;59(1):43-50.e43.

15. Banks AM, Peter ME, Holder GM, et al. Adherence to Disease-Modifying Therapies at a Multiple Sclerosis Clinic: The Role of the Specialty Pharmacist. J Pharm Pract. 2019:897190018824821.

16. Reynolds VW, Chinn ME, Jolly JA, et al. Integrated specialty pharmacy yields high PCSK9 inhibitor access and initiation rates. J Clin Lipidol. 2019;13(2):254-64.

17. Shah NB, Mitchell RE, Proctor ST, et al. High rates of medication adherence in patients with pulmonary arterial hypertension: An integrated specialty pharmacy approach. PLoS One. 2019;14(6):e0217798.

18. Wyatt H, Peter M, Zuckerman A, et al. Assessing the Impact of Limited Distribution Drug Networks Based on Time to Accessing Oral Oncolytic Agents at an Integrated Specialty Pharmacy. Journal of Hematology Oncology Pharmacy. 2020;10(4):198-205.

19. Berger N, Peter M, DeClercq J, et al. Rheumatoid arthritis medication adherence in a health-system specialty pharmacy. The American Journal of Managed Care.

20. Harris PA, Taylor R, Thielke R, et al. Research electronic data capture (REDCap)-a metadata-driven methodology and workflow process for providing translational research informatics support. J Biomed Inform. 2009;42(2):377-81.

21. DeClercq J, Choi L. Statistical considerations for medication adherence research. Curr Med Res Opin. 2020:1-9.
22. Bonafede MMK, McMorrow D, Proudfoot C, et al. Treatment Persistence and Healthcare Costs Among Patients with Rheumatoid Arthritis After a Change in Targeted Therapy. Am Health Drug Benefits. 2018;11(4):192-202.

23. Morgan KP, Muluneh B, Deal AM, et al. Impact of an integrated oral chemotherapy program on patient adherence. J Oncol Pharm Pract. 2018;24(5):332-36.

24. De Vera MA, Mailman J, Galo JS. Economics of non-adherence to biologic therapies in rheumatoid arthritis. Curr Rheumatol Rep. 2014;16(11):460.

25. Marengo MF, Suarez-Almazor ME. Improving treatment adherence in patients with rheumatoid arthritis: what are the options? Int J Clin Rheumtol. 2015;10(5):345-56.

26. Pharmacy Quality Alliance. PQA's Specialty Core Measure Set. https://www. pqaalliance.org/adherence-measures

27. Chatzidionysiou K, Askling J, Eriksson J, et al. Effectiveness of TNF inhibitor switch in RA: results from the national Swedish register. Ann Rheum Dis. 2015;74(5):890-6.

28. Hyrich KL, Lunt M, Watson KD, et al. Outcomes after switching from one anti-tumor necrosis factor alpha agent to a second anti-tumor necrosis factor alpha agent in patients with rheumatoid arthritis: results from a large UK national cohort study. Arthritis Rheum. 2007;56(1):13-20.

29. Chu LH, Kawatkar AA, Gabriel SE. Medication adherence and attrition to biologic treatment in rheumatoid arthritis patients. Clin Ther. 2015;37(3):660-6.e668.

30. Tkacz J, Ellis L, Bolge SC, et al. Utilization and adherence patterns of subcutaneously administered anti-tumor necrosis factor treatment among rheumatoid arthritis patients. Clin Ther. 2014;36(5):737-47.

31. Calvo-Alén J, Monteagudo I, Salvador G, et al. Non-adherence to subcutaneous biological medication in patients with rheumatoid arthritis: a multicentre, non-interventional study. Clin Exp Rheumatol. 2017;35(3):423-30. 